\title{
Neurosonological Diagnosis in the Acute Phase of Stroke is a Sign of Qualified Care
}

\author{
Neurosonology makes the difference in acute Stroke Care
}

\section{(ㄷ) (ㄱ) $($ 우}

Authors

Felix Schlachetzki ${ }^{1}$, Max Nedelmann², Holger Poppert ${ }^{3}$, Dorothee Saur ${ }^{4}$, Andreas Harloff ${ }^{5}$, Jan Liman ${ }^{6}$, Matthias Reinhardt ${ }^{7}$, Erwin Stolz ${ }^{8}$, Manfred Kaps ${ }^{9}$

\section{Affiliations}

1 Klinik und Poliklinik für Neurologie der Universität Regensburg, Klinik für neurologische Rehabilitation, Bezirksklinikum Regensburg, Regensburg

2 Klinik und Poliklinik für Neurologie am Universitätsklinikum Eppendorf, Hamburg, Klinik für Neurologie der Regio Kliniken GmbH Pinneberg

3 Klinik und Poliklinik für Neurologie der Technischen Universität München, München

4 Klinik und Poliklinik für Neurologie am Universitätsklinikum Leipzig, Leipzig

5 Klinik und Poliklinik für Neurologie und Neurophysiologie am Universitätsklinikum Freiburg, Freiburg

6 Klinik für Neurologie der Universitätsmedizin Göttingen, Georg-August-Universität, Göttingen

7 Klinik für Neurologie und klinische Neurophysiologie, Klinikum Esslingen GmbH, Esslingen

8 Gemeinschaftspraxis, Frankfurt

9 Klinik und Poliklinik für Neurologie, Universitätsklinikum Giessen, Giessen

Bibliography

DOI https://doi.org/10.1055/s-0043-109331

Neurology International Open 2017; 1: E182-E188

(C) Georg Thieme Verlag KG Stuttgart · New York

ISSN 2511-1795

Correspondence

Prof. Dr. med. Manfred Kaps

Neurologische Klinik der Justus-Liebig-Universität Giessen

Klinikstr. 33, 35385 Giessen

Germany

manfred.kaps@neuro.med.uni-giessen.de

\section{ABSTRACT}

Ultrasonography of the extra- and intracranial vessels has been an essential part of the approved concept of stroke units in Germany. This review aims to highlight unique selling points of neurosonology in the acute phase of stroke.

In acute ischemic stroke, occlusion or stenosis of an arterial segment induces individually variable and complex changes in cerebral hemodynamics. Acute arterial occlusion may persist, may partially or completely recanalize, re-occlude after initial recanalization or may be compensated by collateral blood flow. Ultrasonography is most suitable to monitor these hemodynamic changes in the early stages of a stroke. The earlier neurosonography is performed, the better the pathophysiological understanding is, and the more targeted are therapeutic efforts to limit acute neurovascular injury. A general view is that CT angiography and/or magnetic resonance imaging and neurosonographical techniques in acute stroke care are competitive and interchangeable. However, these methods are rather complementary since the visualization of blood vessels, cerebral perfusion and hemodynamic flow is based on different physical principles. Each method has particular advantages and limitations. With respect to the specific diagnostic question, one method or the other may be indicated. It is the responsibility of the treating neurologist to select the most appropriate imaging method and to provide specific competence in neurosonography. The main scope of this article is to motivate neurologists to apply neurosonography specifically in the acute phase of stroke in order to get an independent assessment and unique hemodynamic information instead of leaving neurovascular competence to radiologists. Purposeful use of neurosonologic techniques makes the difference in high quality stroke care.

\section{Introduction}

The new guidelines of the German Stroke Society (Deutsche Schlaganfallgesellschaft, DSG) for the treatment of acute stroke and the revision of the Stroke OPS 8-981 and 8-98b, in which neurological ultrasound diagnostics is no longer an obligatory diagnostic procedure if CT or MR angiography is available during treatment in the stroke unit will have a significant impact on standard of care. This article will describe the specific diagnostic potential of neurovas- 
cular ultrasound in the early phase of the cerebral infarction and will present this procedure as an indicator of high quality and competence in neurovascular treatment.

Since five large randomized trials have demonstrated the efficacy of thrombectomy in patients with occlusions of the distal internal carotid artery and the proximal middle cerebral artery, there is a need to rethink stroke treatment in stroke units and neurovascular centers and to organize in stroke networks. While in the past intravenous IV thrombolysis required only a simple clinical neurological score, an appropriate time window, preclusion of contraindications including computed tomography to rule out cerebral hemorrhage, now vascular diagnosis with detection of intracranial vessel occlusion has become a central issue in the diagnosis of acute cases. The revitalized interest in the neurovascular status is advantageous not only for the approx. $5 \%$ thrombectomy candidates, but also benefits all stroke patients, including the approximately $20 \%$ patients with so-called "stroke mimics" diagnosed and treated on our stroke units. With good reason, the 24/7 availability of neurovascular ultrasound diagnosis is deemed "mandatory" by the German Stroke Society for stroke unit certication and was found to be "absolutely" necessary by a majority of international stroke researchers according to a recent survey $[1,2]$.

\section{Basic Parameters of Stroke Diagnostics}

When it comes to treatment of acute stroke, neurovascular sonography is the only diagnostic instrument in the hands of the treating neurologist. Otherwise, the indication for CT and MRI examination can only be formally provided by radiologists or neuroradiologists and relevant specialists. This also applies in particular to the prioritization of examinations in the face of limited capacity. Stroke registry data show that ultrasound diagnostics together with native CT are by far the most common means of diagnosis in routine care. Ultrasound is widely available around the clock at all levels of care, with no additional technical assistance required to maintain 24hour readiness. Administration of contrast agent such as required for CT or MR angiography (CTA or MRA) with attendant risks (allergic reaction, renal failure, hyperthyroidism) can generally be dispensed with, which makes the procedure safer, and the examination can be performed at the patient's bedside. High-quality color duplex sonography equipment is now available for most neurologists, thus providing highly reliable and accurate diagnosis.

\section{Neurosonography, CT or MR angiography?}

In many cases, CTA, MRA and ultrasound are regarded as competing methods in stroke care. In truth, however, these are complementary procedures that produce images representing blood vessels, brain perfusion and blood flow based on very different physical principles. One method or another is indicated, depending on the specific clinical issue. Likewise, 24-hour availability, therapeutic consistency and the ability of the patient to cooperate also play a role. It is up to the clinically responsible neurologist to make the right choice with respect to the therapeutic consequences.

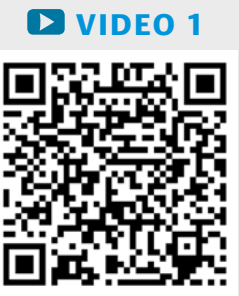

Female patient with a transient ischemic attack. Thrombus in the lumen of the internal carotid artery with ligulate fluttering distal tail. Above the round-oval depiction of the jugular vein.

Morphological imaging using ultrasound is based on the pulse-reflection behavior of tissue. The measurement of flow velocity is based on the Doppler principle, according to which moving objects in a sonic field cause a frequency shift. Color duplex sonography combines both physical principles. It can be deduced from this that morphological features of tissue and blood flow reflect a specific image that is fundamentally different from other imaging methods.

The advantages of imaging methods are clearly shown in the rendering of vessel wall diseases of the brain supplying arteries. Arteriosclerotic plaques, mobile thrombi ( $\nabla$ Video 1 ), inflammation in Takayasu or giant cell arteritis can be displayed with ultrasound in incomparably high spatial and temporal resolution. It is therefore useful to combine different imaging methods to obtain precise information on the pathogenicity of vascular disease or the dynamics of an occlusion process (see below).

\section{Reliability of Neurosonography}

Transcranial color duplex sonography (TCCS) can identify occlusions of the large intracerebral arteries of the circle of Willis with very high sensitivity and excellent specificity $[3,4]$. Occlusion of the main stem of the middle cerebral artery should be assumed, if the ipsi- and contralateral anterior cerebral artery or contralateral middle cerebral artery but not the ipsilateral middle cerebral artery can be displayed. In the absence of a temporal bone window and/ or in order facilitate a rapid and valid diagnosis echo enhancers may be employed [5]. As with any other sophisticated method, ultrasound diagnosis is also examiner-dependent. This also applies to other angiographic procedures, ranging from the experience of the medical technical radiological assistants (MTRA) in the positioning of the patient and the measuring fields, timing of the contrast media bolus, selection of suitable sequences through to assessment of the images by a radiologist who has completed a multi-year specialty fellowship.

\section{"Fast-Track" Examination}

Diagnosis during the acute phase of cerebral infarction takes place under strict time pressure. If vascular imaging using CT or even MRI is not initially possible, a rapid ultrasound examination can determine the further procedure, e. g., whether an examination is necessary with sedation or whether contrast medium should be administered despite renal disease. Concretely, "fast-track" means that 

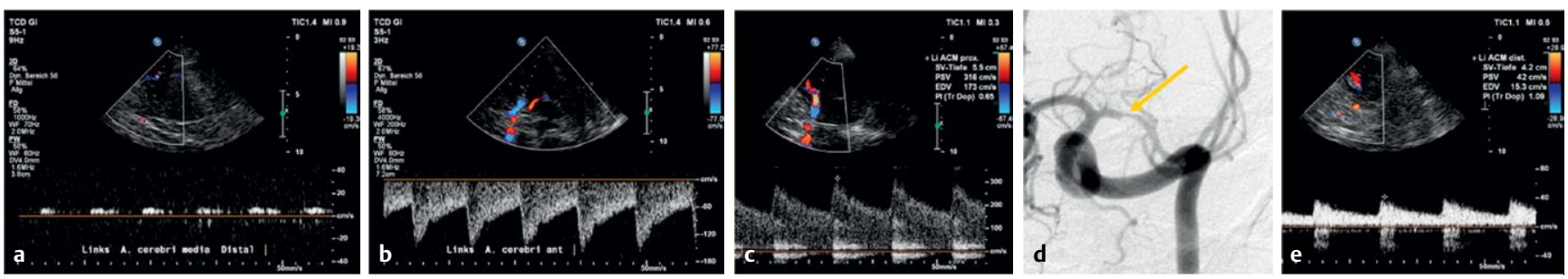

- Fig. 1 Recanalization of a middle cerebral artery: a Occlusion of the middle cerebral artery, $\mathbf{b}$ Flow acceleration in the ipsilateral A1 segment of the anterior cerebral artery is expression of activated leptomeningeal collateralization, $\mathbf{c}$ Four days later high-grade stenosis in the M1 segment of the middle cerebral artery, $\mathbf{d}$ Image obtained using DSA (arrow), e The following day normalization of the flow in the M1 segment..

1) the examination is focused on the symptomatic vascular territory,

2) relevant clinical competence is necessary for planning the steps of the examination,

3) the examination might be performed even after the start of therapy in order to ensure the earliest possible initiation of treatment

4) despite time pressure, emergency diagnostics have to be performed both extra- and intracranially,

5) color duplex sonography is used, which allows an anatomical overview and, in particular, significantly simplifies and speeds up the diagnosis of vessel occlusions. In addition, clear documentation of findings is possible at the push of a button.

6) agitated, confused or aphasic patients can be examined with a little patience.

\section{Ultrasound Findings during the Acute Phase of the Cerebral Infarction}

Each cerebral infarction results from the occlusion of a cerebral vessel. Apart from lacunary infarctions, the occlusion can be more frequently verified the earlier the neurovascular examination is performed. Since the introduction of angiography, it is known that intracranial vessel occlusions can be detected in up to $70 \%$ of collective cases in the first few hours after occurrence, depending on the patient. Ultrasound criteria that correlate with angiography with respect to distal branch occlusions are as follows: occlusions of two or more M2-segments on the affected side and/or if flow velocities in the proximal middle cerebral artery are reduced by more than $21 \%$ ("Zanette index") compared to the unaffected side. It is important to note, that the missing depiction of distal branches of the middle cerebral artery in color mode is not a criterion for branch occlusion.

Recanalizing occlusions are typical findings in the acute phase of ischemic stroke ( $\triangleright$ Fig. 1). In the case of distal middle cerebral artery branch occlusions, during the first hours after symptom onset, spontaneous recanalization takes place, whereas in the case of carotid T or middle cerebral artery main trunk occlusions only a low spontaneous recanalization rate can be expected during this period ( $\triangleright$ Fig. 2) [6, 7]. This implies that highly variable hemodynamic conditions may be present in the acute phase of a cerebral infarction. These are not clinically obvious but can be directly detected by neurosonography. Likewise, fluctuating occlusion processes may be immanent. The described pathophysiological mechanisms explain why ultrasound examinations have to be carried out as soon as possible after the stroke to determine the cause of the

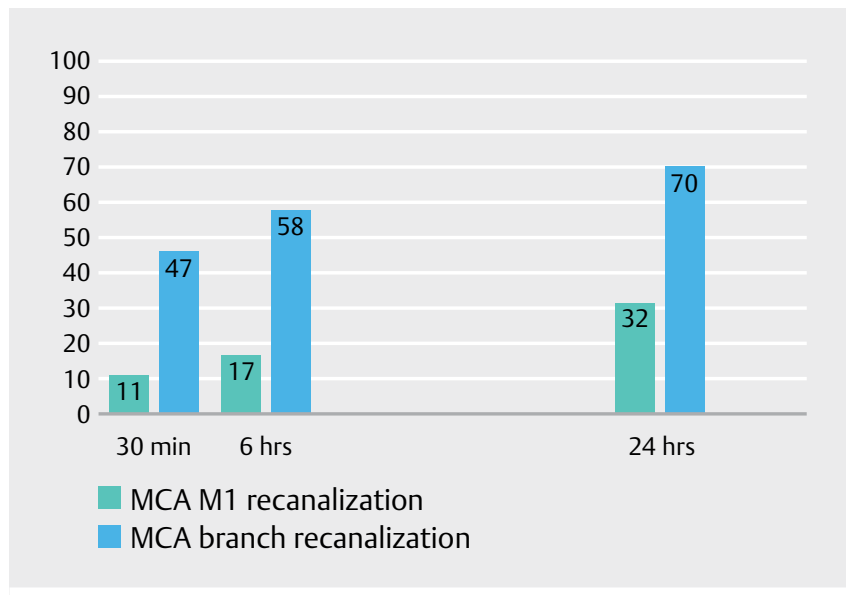

- Fig. 2 Sonographic follow-up examination of 99 patients treated with IV lysis. Recanalization rates (\%) after $30 \mathrm{~min}$, after 6 and $24 \mathrm{~h}$. Branch occlusions (blue) recanalize more frequently than M1 occlusions (green). A late recanalization up to $24 \mathrm{~h}$ was still associated with a better mRS after 3 months compared to non-recanalized cases (better late than never) [6].

event and not at a later time point. Follow-up examinations at a later point in time will then provide additional information regarding the etiology of the stroke, such as identifying changes to the initial findings (spontaneous recanalization generally occurs after embolic occlusions). In addition, the initial vascular examination allows a very early prognostic statement about the further clinical course ( $\triangleright$ Fig. $\mathbf{3}$ ) [8].

Furthermore, symptomatic carotid stenoses must be promptly identified, that is, directly upon the patient's admission, in order to decide upon an intervention as early as possible when planning future treatment. The combination of B-image sonographic plaque characterization to assess embolism risk and Doppler sonographic estimation of hemodynamic changes (by measuring the collateral circulation) explains the pathomechanism of symptomatic internal carotid artery stenoses and can determine early medical secondary stroke prevention [9-11].

\section{Follow-up Examinations in the Stroke Unit and "Stroke Mimics"}

The possibility of performing the examination in real time, simple performance at the patient's bedside, and random repeatability as 
needed, enable TCCS to provide effective monitoring of recanalization therapies and spontaneous changes in findings. The TIBI score (Thrombolysis in Brain Ischemia) and COGIF score (Consensus on Grading Intracranial Flow Obstruction) represent two ultra-

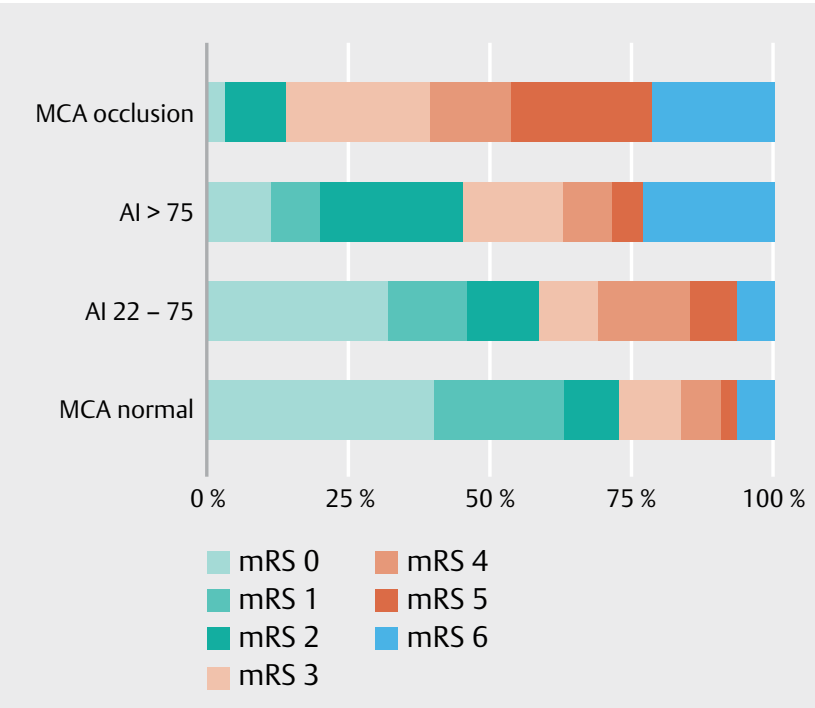

- Fig. 3 In a multicenter prospective NAIS study, 361 patients were sonographically examined extra-and transcranially within $6 \mathrm{~h}$. Of these 121 (34\%) has a normal finding of the middle cerebral artery; $176(48 \%)$ has branch occlusions; 7 (2\%) exhibited high-grade middle cerebral artery stenosis, and 57 (16\%) had an M1 occlusion. There was an almost linear relationship with the clinical condition (mRS) after 3 months: the more branches were closed, the lower the probability of minor disability. A reliable prognostic statement can already be made during intake. (Al = Zanette Asymmetry Index) (modified according to [8]) sound-based scoring methods allowing the quantification of recanalization success as well as determination of the lack of therapeutic success, reocclusion or hemodynamically critical situations in order to respond to them at an early stage ( $\mathbf{F i g . 4 )}[6,12,13]$. In the acute phase a partially recanalized embolus often exhibits the typical findings of intracranial stenosis ( $\bullet$ Fig. 1). A decrease in the degree of stenosis, or a normalization of blood flow conditions in subsequent days distinguishes the symptoms from atherosclerotic stenosis. In the (not uncommon) common case of spontaneous recanalization which has already taken place prior to the initial diagnosis, the detection of functional postischemic hyperemia in the symptomatic vessel section can provide evidence of the underlying pathophysiology.

It is commonly known that the diagnosis of "stroke" often conceals approx. 15-30\% of so-called "stroke mimics". Emergency rooms can treat patients suffering from Todd's paresis after an epileptic attack, migraine attacks with auras, somatization disorders, electrolyte and glucose imbalance or peripheral vestibular disorders. A pathological ultrasound in the "fast track" is an important finding, which can rule out a "stroke mimic" [14].

\section{Mobile Thrombi and Dissection}

In addition to showing the exact location of an ICA occlusion - proximal vs. distal - imaging can provide a more exact assessment of the underlying pathology. The display of a mobile thrombus during recording ( $\square$ Video 2 ) is relevant for further treatment planning and can justify acute intervention in an individual case [1517]. Systematic studies on the best possible treatment of free-floating thrombi are rare because they can only be detected by means of real-time ultrasound and avoid routine diagnosis with CT or MR angiography.
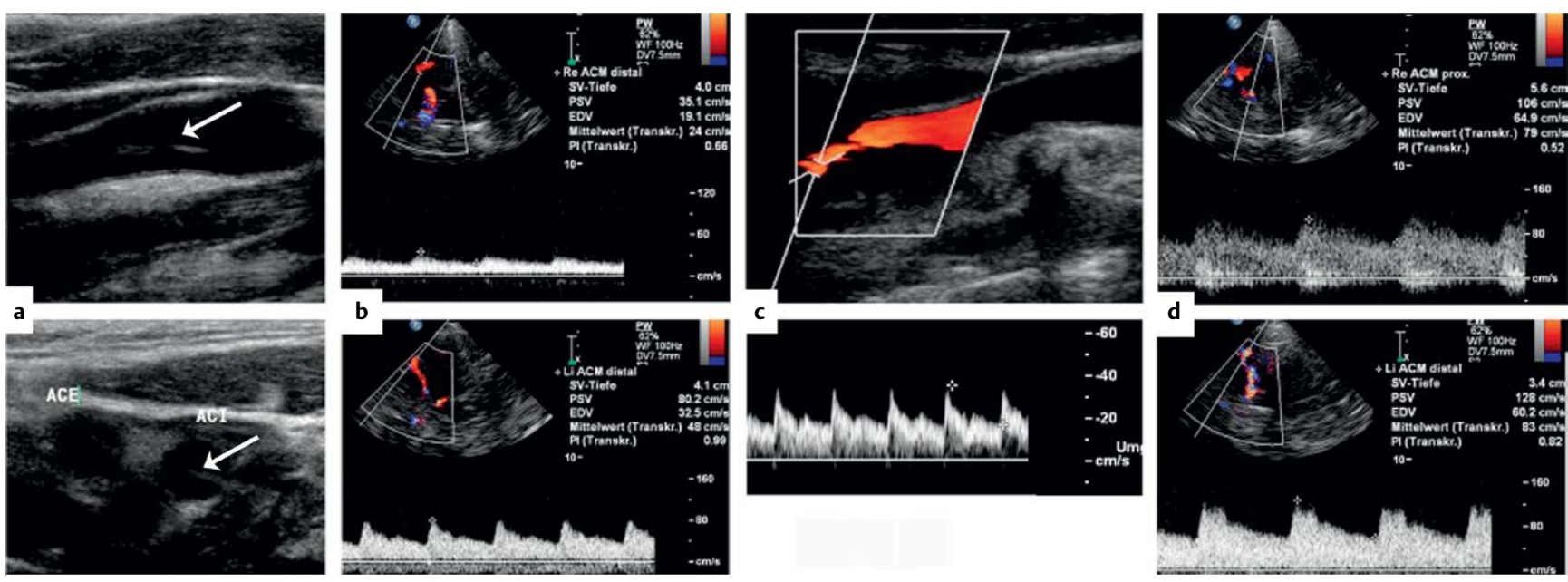

- Fig. 4 A 44-year-old patient reported sudden visual disturbances while swimming. Shortly thereafter a severe hemiparesis of the left side of the body occurred. During inpatient intake approx. $1 \mathrm{~h}$ later he experienced severe media syndrome with hemiplegia and neglect. a Color duplex sonography revealed occlusion of the right internal carotid artery resulting from dissection (white arrow points to dissection membrane, top in longitudinal section, bottom in cross-section). b Hemodynamically reduced perfusion in the right circulatory pathway region with attenuated current pulse curve (top). Left middle cerebral artery with normal perfusion (bottom) absent collateralization. MR tomography showed infarctions in the anterior right border zone. c Follow-up 5 days later showed spontaneous recanalization of the right internal carotid artery. $\mathbf{d}$ Postischemic hyperperfusion syndrome in the right middle cerebral artery after carotid recanalization (top). Normal left middle cerebral artery for comparison (bottom). Initially, a so-called "triple H" therapy was initiated after lysis due to the hemodynamically critical situation; after extracranial recanalization. Due to hyperperfusion the therapy was reversed. 


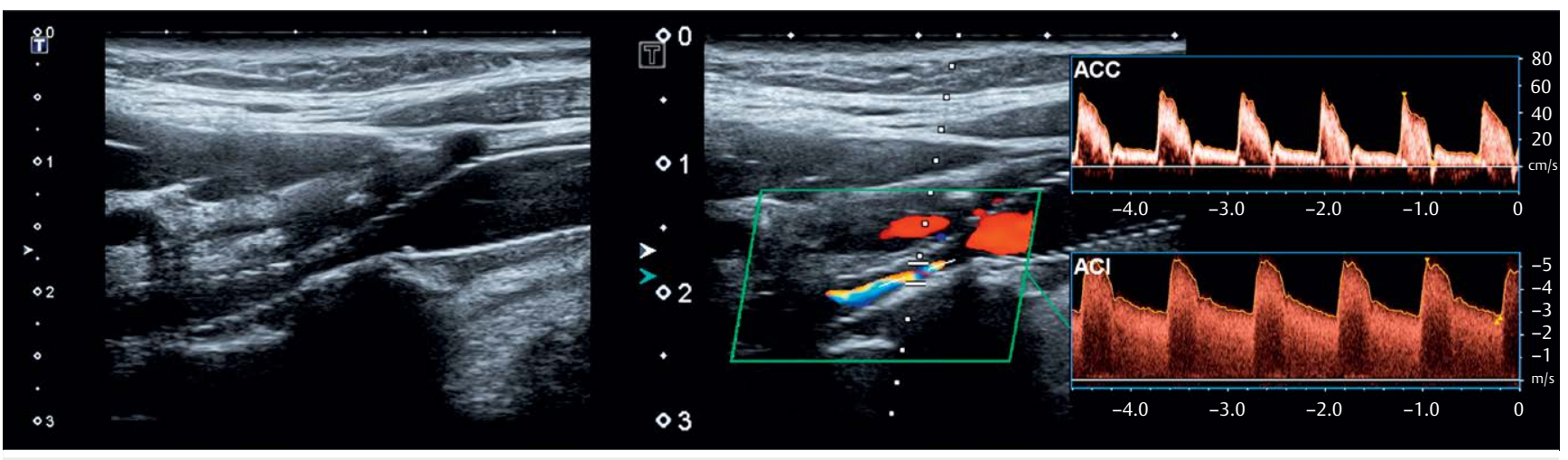

- Fig. 5 In-stent stenosis of the internal carotid artery. a Incomplete unfolding of the stent. b Detection of flow acceleration to $500 / 300 \mathrm{~cm} / \mathrm{s}(\mathrm{sys}-$ tolic/diastolic maximum flow). The relatively low flow disturbances with maintained systolic window and absent low-frequency components indicate that the sole assessment of flow velocities overstate the degree of stenosis of in-stent stenosis.

VIDEO 2

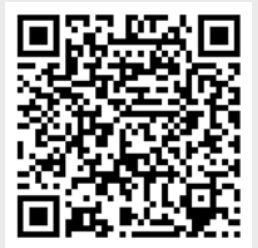

Floating lumen-occluding thrombus in the internal carotid artery (ICA) with a distal (cranial) tale. Due to this finding and about two hours after initial IV thrombolysis anticoagulation with IV heparin was started resulting in complete recanalization and unmasking the ulcerated high grade ICA stenosis (approx. 90\%, NASCET graduation.

In many cases, spontaneous dissection of the large extracranial, cerebral arteries can be determined sonographically by means of unambiguous wall changes in proximal vessel sections $[14,18]$. Dissection at the level of the cranial base, on the other hand, is difficult to diagnose. Signs of increased peripheral flow resistance and poststenotic changes are found intracranially with a heavy lumen obstruction. According to the current update of the guidelines, dual imaging diagnostics using MRI with T1-weighted, fat-suppressed axial sequences and neurovascular ultrasound is still the diagnostic approach of choice, but the importance of CT angiography as a reliable alternative to MRI has increased [19]. Because of the disease dynamics, the early initial examination and further sonographic follow-ups are particularly important in the acute phase (both progression and frequently, recanalization), since dissection of additional arteries or progressive dissections may be detected, thus necessitating individual adaptation of the secondary prophylaxis, blood pressure therapy and continuation of monitoring ( $>$ Fig. 4).

Detection of a proximal dissection of the left common carotid artery (CCA) is easily pathognomically recognized as an aortic dissection, even for a relatively inexperienced examiner [20]. An aortic dissection extending in the common carotid artery is life-threatening, and the patient must be transferred immediately to cardiac surgery.

\section{Inflammatory Cerebrovascular Diseases}

Giant cell arteritis (GCA) is the most common primary systemic vasculitis. Since their predilection sites are the medium-to-large arteries, especially the branches of the external carotid artery, there have been pioneering findings in this regard. Likewise intracranial arteries, especially the vertebral artery, may be affected. As much of the length of the extracranial branches of the external carotid artery as possible should be examined. Hypoechogenic vessel wall edema (halo sign) is pathognomonic with high specificity (97\%). Diagnostic sensitivity is significantly above $80 \%$, thus if a clear ultrasound is obtained without vascular biopsy, steroid therapy should be initiated [21]. In addition, local stenoses (flow accelerations) can be observed, which can also serve as a strong proof of the presence of vasculitis, even without evidence of vascular edema, for example after the onset of a steroid treatment or in the absence of atherosclerosis. In this context, duplex sonography is especially useful in patients with acute visual loss because GCA often affects the posterior ciliary arteries leading to anterior ischemic optic neuropathy (AION) and rarely occludes the central artery. Embolic central artery occlusion as an essential differential diagnosis to (GCA) can be demonstrated in duplex transorbital sonography as a hyperechoic central artery occlusion (so-called spot sign) inside the proximal section of the optic nerve, and appears to be a highly-valid prognostic factor for thrombolytic therapy $[22,23]$. Sonographic representation of the retro-orbital arteries is regarded as the sole highly-specific finding of the method.

\section{Ultrasound Diagnostics after Postinterventional Complications}

Patients with acute symptomatic carotid artery stenosis are often treated in the stroke unit after carotid endarterectomy (CEA) or carotid angioplasty with stent implantation (CAS). In the immediate postoperative ultrasound examination after CEA, it is necessary to detect residual defects and complications such as microsuture ruptures, intimal steps, dissections, pseudoaneurysms, thrombi and remaining stenoses which may require re-intervention [24, 25]. 
Examination conditions are often made more difficult by swelling or hematomas in the area of the surgical wound.

Early complications after CAS are, in particular, an incomplete unfolding of the stent with resulting in-stent stenosis (ISS) ( $\triangleright$ Fig. 5) or even occlusion. In general, during follow-up monitoring, ISS is systematically over-estimated when applying conventional criteria for carotid stenosis (especially systolic peak velocity [PSV] but also end-diastolic velocity [EDV]) [26-28]. Therefore Lal and colleagues suggested modified threshold values based on the ICA/ CCA stenosis index [27]. In addition, the conventional criteria such as pre- and post-stenotic flow changes as well as activation of collateral pathways should be taken into account [29, 30].

The reason for the increased flow rates in the stent-supplied ICA appears to be, in addition to the reduction of vessel diameter after introduction of the stent, reduced arterial compliance [26, 31] and altered geometry of the blood vessel [32]. Longitudinal ultrasound examinations of 82 patients with stent-supplied ICA showed that postinterventional moderately increased flow velocities in the stent (PSV $98.5 \pm 21 \mathrm{~cm} / \mathrm{s}$, EDV $25.5 \pm 8.1 \mathrm{~cm} / \mathrm{s}$ ) remained stable for more than 1 year, contradicting the idea that initial hyperperfusion after CAS causes increased flow velocities [33]. Pragmatically, an early baseline ultrasound examination is recommended in the first days after stent implantation, which can then be used for the follow-up observations in order to quantify later complications such as a hyperplasia of the neointima or formation of neoartherosclerosis.

\section{Discussion}

The impetus for this article was the DSG notification that ultrasound examination during hospitalization in the stroke unit is no longer obligatory for the deduction of complex treatment of stroke according to the DIMDI (Deutsches Institut für Medizinische Dokumentation und Information/German Institute for Medical Documentation and Information) if another imaging method has been used to diagnose the blood vessels supplying the brain. Without a doubt, the absolute need for early vascular diagnostics represents an advance and is indispensable when deciding whether endovascular thrombendarterectomy is required. The new OPS formulation also suggests that methods for neurovascular imaging are interchangeable, and that ultrasound diagnostics can also be performed at a later stage, if not entirely dispensable. The aim of the present article is therefore to again describe the unique features of sonography, and to explain how the patient's individual pathophysiology can be optimally understood, and how sonography continues to provide high value in the care of acute stroke patients. It would be an unfortunate collateral damage if the successfull implementation of endovascular stroke treatment would weaken the well established stroke unit concept by abandoning neurovascular ultrasound.

After the onset of an ischemic stroke, complex, individually different, hemodynamic transformation processes decisive for the fate of the patient are triggered in the affected vessel segment and associated collateral pathways. Occlusions can persist, recanalize partially or completely compensate collaterally or re-occlude after recanalization ( $\vee$ Fig. 2 ). In the initial hours post-onset these processes can be more closely tracked using ultrasound compared to any other method. The sooner the examination is performed, the more revealing are the findings, the individual pathophysiological understanding is more extensive and the benefit for therapeutic decisions is greater.

In the "one-stop-shop" strategy increasingly being used in the daily diagnostic routine in the care of acute stroke cases, instrument-based diagnosis is delegated to neuroradiology while medication-based secondary prophylaxis is handled by internal medicine. Neurosonology makes a difference in this respect: it is widely accepted as a core competency of instrument-based neurology and is considered a characteristic of high-quality stroke treatment. Due to the enormous technological advances in neurology, excellent imaging methods are available in stroke diagnosis which can be indicated as a function of the clinical issue and availability, and which can be used in parallel or as a complement. The demonstration of impressive ultrasound findings parallel to radiological findings in the daily „radiology conference“ would be a useful step to anchor neurosonology in the awareness of neurologists. Whereas CT and MRI diagnostics require years of specialist education, neurologists learn this method formally within the course of normal training. Unfortunately there is decreasing time for this, due to increasing consolidation of subject matter in medical education.

The particular competence in treating stroke cases as claimed by neurologists is ultimately not justified due to the fact that diagnostic requirements are met by other specialties. The aim of this article is therefore to motivate neurologists to deal more intensively with neurosonology in the acute phase of the stroke, and obtain an image themselves, rather than having an image made for them. For the purpose of individualized medicine, neurosonography provides very specific neurovascular information that neurologists should use when discussing complex stroke patients with neuroradiologists, vascular surgeons and cardiologists, and should remain an indispensable component of the successful stroke unit concept.

\section{Conflict of Interest}

No conflict of interest has been declared by the authors.

\section{References}

[1] Leys D, Ringelstein EB, Kaste M et al. The main components of stroke unit care: results of a European expert survey. Cerebrovasc Dis 2007; 23: 344-352

[2] Ringelstein EB, Busse O, Ritter MA. Das Stroke-Unit-Konzept in Deutschland und Europa. Nervenheilkunde 2010; 29: 836-842

[3] Gerriets T, Goertler M, Stolz E et al. Feasibility and validity of transcranial duplex sonography in patients with acute stroke. J Neurol Neurosurg Psychiatry 2002; 73: 17-20

[4] Herzberg M, Boy S, Holscher T et al. Prehospital stroke diagnostics based on neurological examination and transcranial ultrasound. Crit Ultrasound J 2014; 6: 3

[5] Postert T, Federlein J, Przuntek $\mathrm{H}$ et al. Insufficient and absent acoustic temporal bone window: potential and limitations of transcranial contrast-enhanced color-coded sonography and contrast-enhanced power-based sonography. Ultrasound Med Biol 1997; 23: 857-862 
[6] Wunderlich MT, Goertler M, Postert T et al. Recanalization after intravenous thrombolysis: Does a recanalization time window exist? Neurology 2007; 68: 1364-1368

[7] Xu Y, Qian G, Wei L et al. Predictive factors for the spontaneous recanalization of large and middle cerebral arteries after acute occlusion. J Stroke Cerebrovasc Dis 2016; 25: 1896-1900

[8] Allendoerfer ], Goertler M, von Reutern GM et al. Prognostic relevance of ultra-early doppler sonography in acute ischaemic stroke: A prospective multicentre study. Lancet Neurol 2006; 5: 835-840

[9] Shahidi S, Owen-Falkenberg A, Hjerpsted U et al. Urgent best medical therapy may obviate the need for urgent surgery in patients with symptomatic carotid stenosis. Stroke 2013; 44: 2220-2225

[10] Arntzen KA, Schirmer H, Johnsen SH et al. Carotid artery plaque progression and cognitive decline: The Tromso Study 1994-2008. Eur J Neurol 2012; 19: 1318-1324

[11] Markus HS, Droste DW, Kaps M et al. Dual antiplatelet therapy with clopidogrel and aspirin in symptomatic carotid stenosis evaluated using doppler embolic signal detection: The Clopidogrel and Aspirin for Reduction of Emboli in Symptomatic Carotid Stenosis (CARESS) trial. Circulation 2005; 111: 2233-2240

[12] Nedelmann M, Stolz E, Gerriets T et al. Consensus recommendations for transcranial color-coded duplex sonography for the assessment of intracranial arteries in clinical trials on acute stroke. Stroke 2009; 40: 3238-3244

[13] Demchuk AM, Burgin WS, Christou I et al. Thrombolysis in brain ischemia (TIBI) transcranial Doppler flow grades predict clinical severity, early recovery, and mortality in patients treated with intravenous tissue plasminogen activator. Stroke 2001; 32: 89-93

[14] Hand PJ, Kwan J, Lindley RI et al. Distinguishing between stroke and mimic at the bedside: the brain attack study. Stroke 2006; 37: 769-775

[15] Herzig R, Skoloudik D, Kral M et al. Ultrasonographic and perioperative macroscopic findings in acute carotid artery occlusion. J Neuroimaging 2011; 21: 5-9

[16] Weis-Muller BT, Spivak-Dats A, Turowski B et al. Time is brain?-Surgical revascularization of acute symptomatic occlusion of the internal carotid artery up to one week. Ann Vasc Surg 2013; 27: 424-432

[17] Hill SL, Brozyna W. Extensive mobile thrombus of the internal carotid artery: A case report, treatment options, and a review of the literature. Am Surg 2005; 71: 853-855

[18] Vicenzini E, Giannoni MF, Ricciardi MC et al. Noninvasive imaging of carotid arteries in stroke: Emerging value of real-time high-resolution sonography in carotid occlusion due to cardiac embolism. J Ultrasound Med 2010; 29: 1635-1641

[19] Ringelstein EB, Dittrich R, Sitzer M et al. Spontane Dissektionen der extra- und intrakraniellen hirnversorgenden Arterien. Akt Neurole 2016; 43: 418-427
[20] Seliger C, Turmanidze N, Schmid E et al. Three cases of stroke in patients with atypical presentation of type a aortic dissection-potential of neurosonography in the early diagnosis of atypical stroke. Ultraschall Med 2011; 32: 619-621

[21] Schmidt WA, Blockmans D. Use of ultrasonography and positron emission tomography in the diagnosis and assessment of large-vessel vasculitis. Curr Opin Rheumatol 2005; 17: 9-15

[22] Nedelmann M, Graef M, Weinand F et al. Retrobulbar spot sign predicts thrombolytic treatment effects and etiology in central retinal artery occlusion. Stroke 2015; 46: 2322-2324

[23] Ertl M, Altmann M, Torka E et al. The retrobulbar "spot sign" as a discriminator between vasculitic and thrombo-embolic affections of the retinal blood supply. Ultraschall Med 2012; 33: E263-E267

[24] van der Kolk AG, de Borst G], Jongen LM et al. Prevalence and clinical consequences of carotid artery residual defects following endarterectomy: a prospective $\mathrm{CT}$ angiography evaluation study. Eur J Vasc Endovasc Surg 2011; 42: 144-152

[25] Budincevic $\mathrm{H}$, Ivkosic A, Martinac $\mathrm{M}$ et al. Asymptomatic dissecting intimal lesions of common carotid arteries after carotid endarterectomy. Surg Today 2015; 45: 1227-1232

[26] Lal BK, Hobson RW 2nd, Goldstein J et al. Carotid artery stenting: Is there a need to revise ultrasound velocity criteria? J Vasc Surg 2004; 39: 58-66

[27] Lal BK, Hobson RW 2nd, Tofighi B et al. Duplex ultrasound velocity criteria for the stented carotid artery. J Vasc Surg 2008; 47: 63-73

[28] Nederkoorn PJ, Brown MM. Optimal cut-off criteria for duplex ultrasound for the diagnosis of restenosis in stented carotid arteries: Review and protocol for a diagnostic study. BMC Neurol 2009; 9: 36

[29] Arning C, Widder B, von Reutern GM et al. [Revision of DEGUM ultrasound criteria for grading internal carotid artery stenoses and transfer to NASCET measurement]. Ultraschall Med 2010; 31: 251-257

[30] von Reutern GM, Goertler MW, Bornstein NM et al. Grading carotid stenosis using ultrasonic methods. Stroke 2012; 43: 916-921

[31] Hakimi M, Knez P, Lippert M et al. Altered in-stent hemodynamics may cause erroneous upgrading of moderate carotid artery restenosis when evaluated by duplex ultrasound. J Vasc Surg 2012; 56: 1403-1408

[32] Kamenskiy AV, Pipinos II, Dzenis YA et al. Effects of carotid artery stenting on arterial geometry. J Am Coll Surg 2013; 217: 251-262

[33] Kim ES, Sun Z, Kapadia S et al. Characteristics of duplex sonographic parameters over time after successful carotid artery stenting. J Ultrasound Med 2012; 31: 1169-1174

[34] Kaps M, Stolz E, Allendoerfer J. Prognostic value of transcranial sonography in acute stroke patients. Eur Neurol 2008; 59 (Suppl 1): 9-16 